\title{
Conceptualizing the Pathways of Literature Review in Research
}

Nabaraj Neupane (Corresponding author)

Department of English Education, Prithvi Narayan Campus, Tribhuvan University, Nepal

Email: nabaraj.neupane@prnc.tu.edu.np

Received: 26/08/2020

Accepted: 19/12/2020

Published: 01/01/2021

Volume: 2 Issue: 1

How to cite this paper: Neupane, N. (2021). Conceptualizing the Pathways of Literature Review in Research. Journal of Practical Studies in Education, 2(1), 1-7

DOI: https://doi.org/10.46809/jpse.v2i1.16

Copyright $\odot 2020$ by author(s) and Global Talent Academy Ltd. This work is licensed under the Creative Commons Attribution International License (CC BY 4.0).

http://creativecommons.org/licenses/by/4.0/

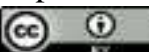

\begin{abstract}
Since research work is an adventurous expedition, one requires clear pathways, without which the researcher can be lost in the foggy way. To develop a good roadmap is, thus, a crucial task on the part of the researcher. Based on this assumption, this study aimed to conceptualize the notions, functions, and components of literature review that subsumes developing a conceptual framework, an outline of a research work. To achieve this objective, I adopted descriptive-interpretive design of research. Basing my study on theoretical and conceptual insights from different theoreticians' and researchers' works, I analyzed and interpreted the information obtained from the templates used by the previous researchers. I have inferred that literature review, which is indispensable in research process, functions like a beacon light for safe harbouring the research work. I have also inferred, like many have done, that the basic components of literature review consist of introduction, review of theoretical and empirical literature, implication of the review, and theoretical and/or conceptual framework/s. Its implication is that any research work needs to pave its pathways distinctly for its successful execution.
\end{abstract}

Keywords: Concept, Framework, Review, Roadmap, Theory

\section{Introduction}

Conceptualizing literature review (LR) is at the centre of discussions in the field of research because LR provides the researcher with guidelines for conceptualizing a research problem, designing suitable methodology, and devising tools/ways of analysis/interpretation of the results. This is evidenced in these words, "The literature review is an integral part of the entire research process and makes a valuable contribution to almost every operational step" (Kumar, 2006, p. 30). Similarly, LR provides justification for the study, pinpoints niche in the existing studies, and proves that the researcher has prepared needed groundwork in research (Bui, 2014). Therefore, writing, editing, and re-editing LR begins when a researcher begins his work and "continues up to the point where you have finished discussing your findings" (Bitchener, 2010, p. 59). This confirms that until a researcher ends up the work, LR continues.

LR refers to the review of the available literature. However, it is not simply a summary of all the available literature, neither is it only a summary of what is read (Bui, 2014). Therefore, Taylor (n. d.) is right to define LR as "an evaluative report of information found in the literature related to your selected area of study". While reviewing, one should summarize, describe, make a judgment, synthesize, conclude, and compress the ideas/views/thoughts/major findings in a prescribed 
format. In a nutshell, LR is a critical survey of academic and scholarly references, such as articles, journals, books, research reports, seminal papers, important documents, and other sources (printed and/or electronic), which are relevant to the study area selected by the researcher. The aims/purposes/functions/uses of LR are many. For Kumar (2006, p. 30), two broad functions are: (a) to provide a theoretical background to your study; and (b) to enable you to contextualize your findings concerning the existing body of knowledge in addition to refining your methodology". However, Bitchener (2010) has presented seven functions. Synthesising these and using my insight into the field for two decades, I have indicated 10 LR functions (Box 1).

\section{Box1: Functions, Roles, or Uses of Literature Review}

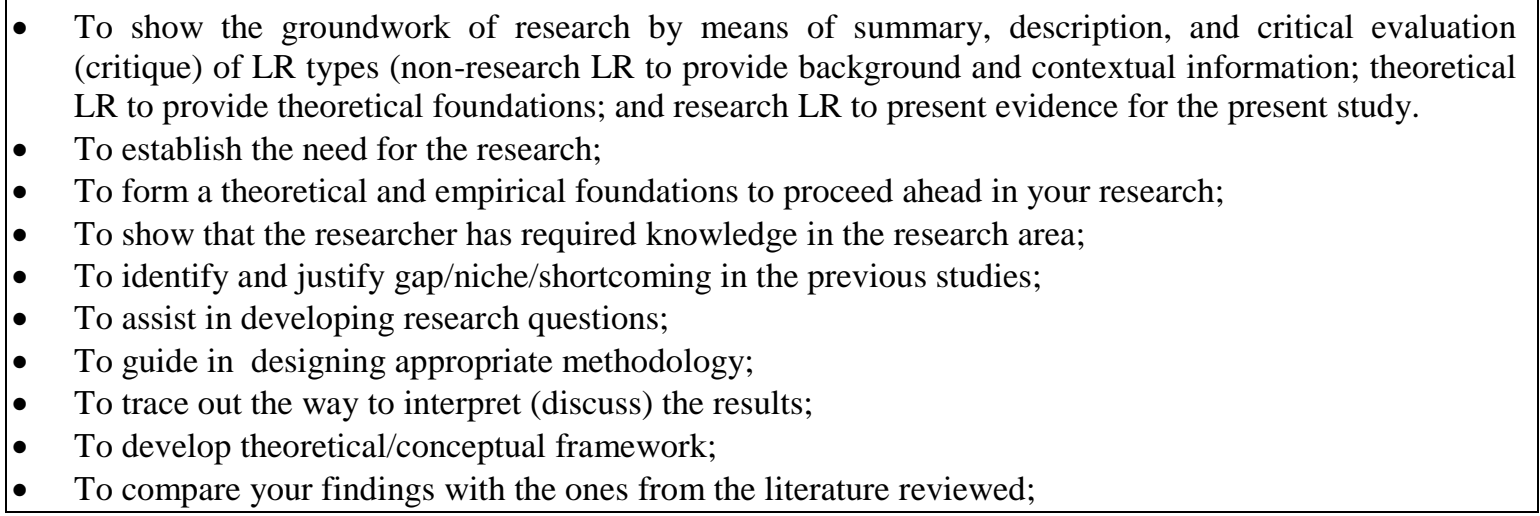

Box 1 shows that the role and place of LR in research work is vital. The review of non-research literature exhibits the background and the context of the study. It also presents the rationale and significance of the study. LR prepares the ground for the researcher employing summarizing, describing, synthesizing, and criticizing the relevant literature available and selected by the research. Therefore, LR forms strong theoretical foundations and empirical evidences for the research work. It also justifies that the researcher has obtained required knowledge base in the study area. This also convinces the readers that the researcher has developed fundamentals of the study. Synthesizing and criticizing literature enables the researcher to notice and explore gaps and shortcomings in the previous studies; and helps to justify that the present research is new and different from others. Then, the researcher should conceptualize research problems and develop questions. This what-aspect necessitates the ways to seek answers. In this way, LR provides guidelines to design a suitable research paradigm for collecting data, analyzing and interpreting the results. For all these, the researcher is pushed forward to develop a theoretical/conceptual framework. Finally, LR assists the researcher to compare and contrast the present findings with those of others' studies. Thus, LR is vital in each operational step of the research process.

These delineations give me an impetus to set the objectives of the present article, which investigates the term literature review, its concepts, functions, and structures/ components like the review of related theories, previous studies, implications of the review, and the development of theoretical/conceptual frameworks.

\section{Methodology}

This study follows the descriptive-interpretive design, which is used to describe and interpret the templates frequently used by the researchers. This research, thus, is based on secondary research that "includes any research based on secondary sources, especially other researchers' books and articles" (Brown \& Rodgers, 2014, p. 10). The theoretical and conceptual insights that comprise affordability of literature review in research are taken from references like Kumar (2006), Sealey (2010), Bitchener (2010), Bui (2014), and Neupane (2017) among others. Against these insights, the researchers' uses of templates are presented for presenting illustrations and interpretations of the results.

\section{Results and Discussions}

Like in other academic writings, LR Chapter constitutes these three components: introduction, body, and conclusion. The first part functions like a mirror that reflects the second part, i.e. body, and the last part reiterates concisely what is elaborated in the middle one.

3.1. Introduction

The first part of LR, as in other chapters, is the introduction that informs about a new chapter. This part is written for supplying with a precise overview of the chapter (Bitchener, 2010). It is written in two paragraphs to show connections with the previous chapter and to provide an outline of the chapter, being presented. The former function is recognized by 
"purposeful redundancy", which is placed in each chapter in the beginning, is an intentional reiteration of the main points. It is required to show the seamless connection between/among the parts/chapters of the thesis (Bui, 2014, p.124).

Box 2: Purposeful Redundancy to Begin a Chapter.

This chapter reviews the related theoretical and empirical literatures for the purpose of developing a conceptual framework, which is facilitative of seeking answers to the research questions developed in Chapter One.

Box 3: Advance Organizer in LR Chapter

The first section deals with theoretical perspectives on the area selected. It caters for definition, history,
gap, and technique of translation. The second section presents a critical review of empirical research
for providing evidence that the area selected is significant. The third section highlights the implications
of the review of the study. Finally, based on the review of related literature, a conceptual framework is
developed to address the research problems.

Box 2 demonstrates the close connection between Chapter Two with Chapter One. It also exhibits what constitutes the present chapter precisely. The next function is referred by "advance organizer", the next paragraph, which is a framework of LR chapter, so informs what will be discussed in the chapter (Bui, 2014, p.124). This part illustrates the section-wise synopsis of the chapter (Box 3). If readers do not have enough time to go thoroughly, they can just get information from the advance organizer and skip the chapter. Otherwise, they can be motivated to read in detail.

3.2. Body of the Review

This is the main part of the chapter, which incorporates reviews of non-research or theoretical literature, empirical literature, implications of the review, and the development of a theoretical/conceptual framework. Therefore, the body of the review is the heart of this chapter.

\subsubsection{Review of Theoretical Literature (RTL)}

RTL caters for conceptual literature available in books, journals, articles, archive (historical documents, governmental records), website materials, Internet sources, audio/visual materials, CD/DVD/TV episodes, and so on. However, the main sources of theoretical literature are books and journals which have their limitations. For Martin (1985 as cited in Kumar, 2006, p. 33), in books, findings are, "integrated with other research to form a coherent body of knowledge." However, books may not provide up-to-date information, which journals do (Kumar, 2006).

RTL, which includes definitions and relevant theories, offers the researcher with academic/theoretical foundations to the present research. It also provides a researcher with a set of theories, concepts, and variables, which are in deemed need for the development of the theoretical/conceptual framework.

\subsubsection{Review of the Empirical Literature (REL)/Previous Studies}

REL incorporates research reports, research/research-based articles, theses (Master's), and dissertations (PhD). Review of previous studies provides evidence and convincing background in/for research. As a section in LR Chapter, it includes these topics in "research synthesis" (Bui, 2014, p. 125), which includes (summary, analysis, and critique): (a) Researcher, date, and title, (b) Main objectives or research questions (one or two), (c) Methodology in brief (study design, study area, participants, sampling, sampling strategy, intervention if any, data analysis procedures, and so on), (d) Major findings (maybe one or two), (e) Discussion (interpretation of the results), and (f) Comparison with other studies (researcher's critique) (Sealey, 2010, Bitchener, 2010, \& Bui, 2014).

\section{Box 4: A Sample of Reviewing Empirical Literature. Source: Adapted from Lamichhane (2012)}

Among the studies that focus on translating culture-specific terms, Neupane (2012) in his research,
entitled, "Translation of Culture-Bound Terms in Muglan" has attempted to trace out the strategies
used in translating culture-bound terms. He has collected 220 cultural terms by using non-random
judgmental sampling procedure. He found that the terms, categorized into five types, employed seven
strategies. The most frequent strategy was the translation by cultural substitution $(29.54 \%)$ followed by
loan words (plus explanation) $(29.09 \%)$, neutral word $(16.36 \%)$ and general word (14.09\%). Then, he
also found that translation by paraphrase (a related word) was highly used than paraphrase (unrelated
word) and omission. This study has presented a good account of culture-specific terms and strategies
for translating them. However, its data are limited and they are not sufficient to draw conclusions.

The present example (Box 4) consists of six sentences. The first gives the researcher's surname, year of publishing the research report, title or the research, and main objective. The second sentence presents the methodology in brief, which 
includes sample population, and sampling strategy. The third and fourth sentences show major findings and discussion. The last two sentences are the reviewer's critique of the study.

3.2.3. Implications of the Review for the Study (IRS)

The implication is anything (content, methodology, ways of discussion, or relation of the LR to your research) indirectly suggested/stated. The implication, therefore, refers to the potential uses of LR for the present research. It subsumes the uses, adaptations, or adaptations of the theoretical, empirical, or methodological literature. Box 5 shows the niche in previous studies and justifies the rationale of this research. The researcher received methodological insights by reviewing the literature. IRS revolves around these two questions: (a) How are your reviews useful for your research? (b) How is your research different from the others'? (See Box 5 for clarity).

\title{
Box 5: Sample Implication of the Review for the Present Research Source: Adapted from Bhusal (2015).
}

\begin{abstract}
Through the intensive study of the aforementioned and other related literature, I pinpointed the fact that previous research missed the word level equivalence in terms of syntactic, semantic and pragmatic strategies in the translated version of the novel 'Phülko Attãka'. Thus, the review of the related literature made me feel the need to carry out this study. At the same time, after reviewing all those research works, I developed a concept on the research process and methodological tools which are very beneficial to my research work.
\end{abstract}

\subsubsection{Theoretical framework (TF) and Conceptual Framework (CF)}

For some scholars, TF and CF are conceptual synonyms whereas for others they are "two different constructs" (Imenda, 2014, p. 185). Whether same or different, TF/CF is pivotal in research work since it is facilitative of formulating research questions and designing appropriate methodology. It is a roadmap which traces out avenues of investigation for a researcher. For comparing and contrasting them, terms like research, theory, concept, and framework should be clarified. De Vous et al. (2005) views research as a "systematic, controlled, empirical, and critical investigation of [natural/social] phenomena, guided by theory and hypotheses about the presumed relations among such phenomena" (as cited in Imenda, 2014, p. 186). This view confirms that every research is guided by a particular theory and therefore theory directs the right path to follow in research. This also shows that TF is deemed a necessity to test hypotheses, which are components of experimental research. So, quantitative/deductive (i.e. experimental) studies call for TF. However, some scholars like Smith and Liehr (1999) argue for the need of CF, not TF in generative research which attempts to generate to develop theoretical insights based on the data collected. So, for grounded or qualitative studies, CF is developed to present an outline of the research. Yet, CF stems from TF.

Likewise, a theory has been defined as "a set of interrelated concepts [...] for the purpose of explaining or predicting" and "a blueprint [...] depicts the elements of a structure" (Liehr \& Smith, 1999, p. 8). Thus, a theory is employed to explain and predict the related concepts, terms, and variables in the structural framework of research. So, the theory provides a skeleton of the research and the researcher's task is to fill blood and flesh of claims and evidences. On the other hand, the concept has been defined as "an image or a symbolic representation of an abstract idea" (Liehr \& Smith, 1999, p. 7), whereas Chien and Kramer (1999) view concept as a theory component which "convey the abstract ideas within a theory" (as cited in Liehr \& Smith, 1999, p.7). Hence, theory and concept are associated in such a way that the former opens the floor for the latter and the latter plays on the opened floor for seeking answers for the research questions.

The next term 'framework' is a structure which provides "guidance for the researcher as study questions are fine-tuned, methods for measuring variables are selected, and analyses are planned" (Liehr, \& Smith, 1999, p. 13). That's why, framework in research provides the researcher in the right direction for the exploration of answers to the research questions, appropriate methodology, and analysis procedures. Further, the researcher can reflect on the framework against his/her findings to find out similarities and discrepancies.

Both TF and CF are epistemological/methodological paradigms (i.e. quantitative, qualitative, or mixed). The two are similar in terms of purpose, in which they provide the right direction to the researcher for the investigation of an issue raised. They differ in terms of conceptual meaning, methodology, and the scope of application (Imenda, 2014). Therefore TF, which is the application of a theory the researcher selected, is pre-established generalization implemented to seek answers deductively. So, the researcher solely depends on a selected theory. On the other hand, CF is mapping up of all the concepts/variables synthesized inductively from theoretical and empirical literatures. In short, TF is a particular theory and is a pre-established assumption; whereas CF is a synthesis of the relevant concepts/variables and is developed by the researcher. Similarly, TF has broader application even in other works whereas CF is limited to the particular research. Therefore, TF is usually assigned in traditional (i.e. quantitative) paradigm whereas $\mathrm{CF}$ is developed in qualitative or mixed paradigm.

$\mathrm{CF}$ is not the steps of research, like Title, Objectives, Methodology, Results, and Discussion. It is a written or visual presentation that "explains either graphically, or in narrative form, the main things to be studied - the key factors, concepts, or variables - and the presumed relationships among them" (Miles \&Huberman, 1994, p. 18). CF should be better presented in graphic/pictorial form (flow chart, tree diagram, shape-based diagrams, like triangles, concentric rings, and circles), based 
on the concepts developed by the researcher after reviewing related literature (theoretical, empirical, and methodological). CF is developed from TF, so the former is a part of the latter (Kumar, 2006). Therefore, the relationship between the two is of hyponymy (whole-part relationship). Any part of TF can be subsumed in CF because TF incorporates all the related theories but $\mathrm{CF}$ is synthesized from the theories based on which research problem is based (Kumar, 2006). For example, all the translation techniques, such as Newmark (1988), Venuti (2004), Bassnett (2005), Baker (2018), among others, can fall under TF and only Baker's (2018) taxonomy can be subsumed into CF for the study which aims to explore techniques and gaps in a translated text. Based on the theory the researcher reviews, a CF is borrowed or developed. If borrowed, it can be either adopted (with modification) or adapted (taken as the original one without any modification). Based on this delineation, I have presented a TF (Box 6 and CF Figure 1) for illustrations and interpretations. Box 6 is a pre-established generalization and the researcher tests this theory against the data collected. The researcher depends solely on Newmark's (1988) model to analyze and interpret the results to conclude. On the other hand, Box 6 is developed inductively by the researcher collecting different concepts/variables from different theories like phases of microteaching, related domains like peer teaching, student teaching, arguments for and against microteaching, and so on.

\section{Box 6: A Sample of TF.}

\begin{tabular}{|c|}
\hline 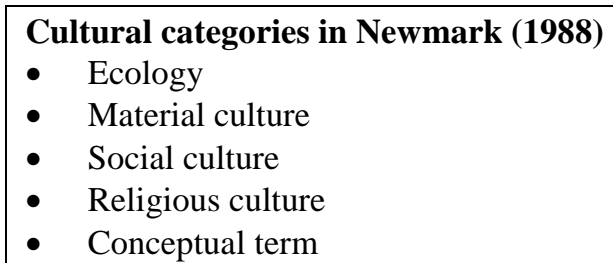 \\
\hline
\end{tabular}

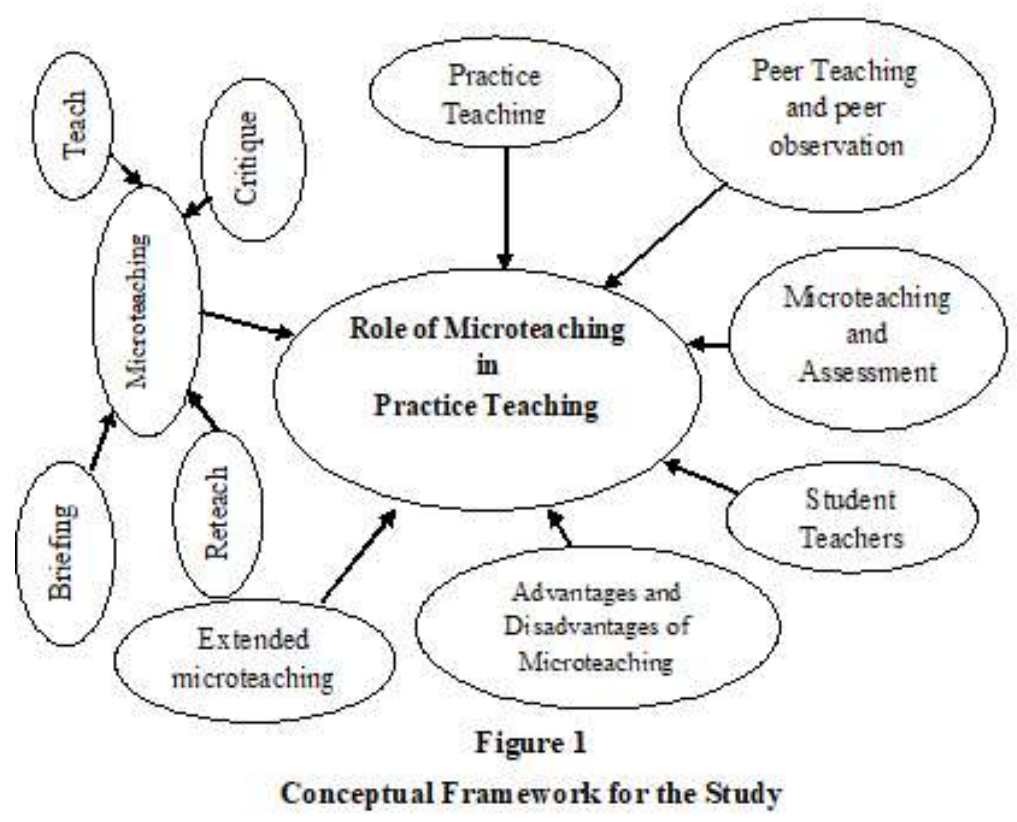

\subsection{Chapter Summary/Conclusion}

A good summary of the main points arouses the reader's "lasting impression" (Bui, 2014, p. 127) and establishes the necessity of the study. In LR Chapter, conclusion recapitulates and synthesizes precisely the key claims. It is the conclusion of the reviewed literatures which sets research paradigm/design to decide appropriate methodology, and opens up the further avenues for analysis and interpretation of the results (Box 7).

\section{Box 7: A Template of LR Chapter Summary}

This chapter has reviewed the related literature in terms of theory and applications and ultimately has developed a conceptual framework. 
Writing LR is not only summing up of the literatures reviewed but also synthesizing them. For achieving the purpose, a researcher should take some considerations (Box 8), which provide them with a set of guidelines to follow while writing a research proposal or thesis.

\section{Box 8: Tips for Writing LR}

- Use claim and evidence structure;

- Be selective (select only the relevant/related /most significant sources for review);

- Use quotes sparingly (better use paraphrasing strategy);

- Be aware of proper citations, i.e. quotations and paraphrasing (see APA Manual, $6^{\text {th }}$ edition, 2010 for detail);

- Keep your voice in reviewing other's works by providing your opening and closing words, views, and ideas;

- Summarize and synthesize the works for review. To summarize is to recapitulate the important information of the sources. To synthesize is to re-organize/re-shuffle the information with adaptations that fit the present research.

- Maintain the assigned chapter structure: Chapter Introduction, Body (RTL, REL, IRS, TF/CF), and Chapter Summary;

- Beware of plagiarism and self-plagiarism;

- If there are headings and sub-headings, use their five levels, do not present numerical sub-headings (see APA Manual, $6^{\text {th }}$ edition, 2010 for detail).

\section{Conclusion}

Literature review (LR) is important since it presents an outline for conducting research work. It is facilitative of each of the operational steps. It ends up only when the research work completes. Thus, LR is an intrinsic part of a whole research process. It prepares the ground for research by acquainting the researcher with the recess/alcove in the others' studies and assist in developing linguistic and/or conceptual framework(s), which in turn guide/s the researcher in designing befitting methodology and congruous ways of analysis and interpretation to draw inferences.

Literature Review Chapter, in the research proposal or thesis, comprises three interrelated parts such as introduction, body, and conclusion. The introduction subsumes purposeful reiteration and an organizer, both of which present a brief outline of the chapter. Body of the LR presents a review of theoretical and empirical literature to lead to a framework, which directs to proceed ahead in the research process. The conclusion also labelled as chapter summary epitomizes and concocts the delineations of the chapter. Thus, LR lies to the heart of the research work that offers the distinct pathways for the researcher to take.

\section{References}

Adhikari, Y. N. (2017). Role of microteaching in practice teaching. English Language Teaching VISION, 3 , 70-82.

American Psychological Association (2010). Publication manual of the American Psychological Association (6 ${ }^{\text {th }}$ ed.). Washington, DC: The Author.

Baker, M. (2018). In other words: A course book on translation ( $3^{\text {rd }}$ ed.). London \& New York: Routledge.

Bassnett, S. (2005). Translation studies ( $3^{\text {rd }}$ ed., $1^{\text {st }}$ Ind. repr.). London \& New York: Routledge.

Bhusal, S. P. (2015). Word level equivalence in the translation of Phulko Atanka. An unpublished M. Ed. Thesis, Tribhuvan University, Nepal.

Bitchener, J. (2010). Writing an applied linguistics thesis or dissertation: A guide to presenting empirical research. UK: Palgrave Macmillan.

Brown, J. D., \& Rodgers, T. S. (2014). Doing second language research. Oxford: Oxford University Press. First published 2002.

Bui, Y. N. (2014). How to write a master's thesis (2 $2^{\text {nd }}$ ed.). Los Angeles: Sage.

Imenda, S. (2014). Is there a conceptual difference between theoretical and conceptual frameworks? Journal of Social Science, 38 (2), 185-195.

Kumar, R. (2006). Research methodology ( $2^{\text {nd }}$ ed.). Delhi: Pearson Education.

Lamichhane, S. (2012). . Translation of sentences: A case of novel Saniko Sahas. An unpublished M. Ed. Thesis, Tribhuvan University, Nepal.

Liehr, P., \& Smith, M. J. (1999). Middle-range theory: Spinning research and practice to creative knowledge for the new millennium. Advance in Nursing Science, 21 (4), 81-91.

Miles, M. B., \& Huberman, A. M. (1994). Qualitative data analysis. London: Sage. 
Neupane, N. (2017). Strategies used in translation of culture specific concepts: Exploration into Nepali Novels (unpublished doctoral dissertation). Tribhuvan University, Kathmandu, Nepal

Newmark, P. (1988). A textbook of translation. London: Prentice Hall.

Sealey, A. (2010). Researching English language: A resource book for students. London \& new York: Routledge

Smith, M. J., \& Liehr, P. (1999). Attentively embracing story: A middle-range theory with practice and research implications. Reseach and Theory for Nursing Practice, 13 (3), 187-204.

Taylor, D. (n. d.). The literature review: A few tips on conducting it. Retrieved from http://www.writing.utoronto.ca/advice/specific -types-of-writing/literature-review.

Venuti, L. (2004). The translation studies reader (e-version). New York: Routledge. 